\title{
A NOTE ON NORMAL DIVISION ALGEBRAS OF PRIME DEGREE*
}

\author{
A. A. ALBERT
}

Wedderburn has proved $\dagger$ that all normal division algebras of degree three over a non-modular field $\Re$ are cyclic algebras. It is easily verified that his proof is actually correct for $\Re$ of any characteristic not three, and I gave a modification of his proof $\ddagger$ showing the result also valid for the remaining characteristic three case. Attempts to generalize Wedderburn's proof to algebras of prime degree $p>3$ have thus far been futile, and it is not yet known whether there are any non-cyclic algebras of prime degree. One notes that in both Wedderburn's proof and my modification one starts by studying a non-cyclic cubic field and thus a subfield of a normal splitting field of degree six with a quadratic (cyclic) subfield. I have generalized this property to the case of arbitrary prime degree and have now provided a new proof of the Weddenburn theorem for algebras of degree three in the characteristic three case. The result is the special case $p=3, m=2$ of the following theorem:

THEOREM. Let $\mathfrak{D}$ be a normal division algebra of degree $p$ over a field $\Re$ of characteristic $p$, and let $m$ be prime to $p$. Then if $\mathfrak{D}$ has a normal splitting field $\mathfrak{B}$ of degree pm over $\Re$, with a cyclic subfield $\mathbb{R}$ of degree $m$ over $\mathfrak{\Omega}$, it follows that the algebra $\mathfrak{D}$ is a cyclic algebra.

In our proof we shall use the following known theorems $\S$ on normal division algebras $\mathfrak{D}$ of degree $n$ over arbitrary fields $\mathfrak{\Omega}$ :

Lemma 1. Let $\mathfrak{R}$ have degree prime to $n$. Then $\mathfrak{D}_{\mathfrak{l}}$ is a division algebra.

Lemma 2. Let $\bigcap_{0}$ have degree $n$ over $\Re$ and split $\mathfrak{D}$. Then $\bigotimes_{0}$ is equivalent to a (maximal) subfield of $\mathfrak{D}$.

Lemma 3. Let $\mathfrak{D}$ have a cyclic subfield of degree $n$. Then $\mathfrak{D}$ is a cyclic algebra.

* Presented to the Society, April 8, 1938.

† Transactions of this Society, vol. 22 (1921), pp. 129-135.

‡ Transactions of this Society, vol. 36 (1934), pp. 388-394.

$\S$ Cf. Deuring's Algebren for our notation and the proofs of the results of Lemmas $1,2,3$. Lemma 4 was proved by the author for $\Re$ of characteristic not $p$, Transactions of this Society, vol. 36 (1934), pp. 885-892, and for $\Re$ of characteristic $p$, ibid., vol. 39 (1936), pp. 183-188. 
Lemma 4. Let $\mathfrak{D}$ of prime degree $n=p$ over $\Re$ have a splitting field $\mathfrak{V}=\mathfrak{\Omega}(y)$, such that $y^{p}=\gamma$ in $\mathfrak{\Omega}$. Then $\mathfrak{D}$ is a cyclic algebra.

To make our proof we let $\$$ be the automorphism group of $\mathfrak{B}$ over $\Re$ and $\mathfrak{S}$ the subgroup of $\mathfrak{S}$ corresponding to $\mathfrak{R}$. Then $\mathfrak{S}$ is a normal divisor of $\mathbb{S t}$ and is of prime order $p ; \mathfrak{S}=[S]$ is a cyclic group. The

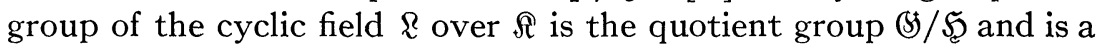
cyclic group $[\mathfrak{S} T]$. Here $T$ is an automorphism of $\mathfrak{S}^{t}$ and $T^{m}=S^{\alpha}$ in $\mathfrak{S}$. But then $\left[\mathfrak{S} T^{p}\right]=[\mathfrak{S} T]$ since $p$ is prime to $m,(\mathfrak{S} T)^{p}=\mathfrak{S} T^{p}$, and $T^{p m}=S^{p \alpha}=I$. Hence we may assume without loss of generality that $T^{m}=I$. Since $\mathfrak{S} T$ has order $m$ so does $T$. The cyclic subgroup $\mathfrak{T}=[T]$ of $(B)$ corresponds to a subfield $乃_{0}$ of degree $p$ over $\Omega$ of $\mathfrak{W}$, and we have the following lemma:

LEMмA 5. The field $\bigcap_{0}$ splits $\mathfrak{D}$.

For clearly $\mathfrak{W}$ is the composite of $\mathfrak{Z}_{0}$ and $\mathfrak{R}$, and $\mathfrak{W}=\left(\mathfrak{B}_{0}\right)_{\mathfrak{R}}$. Now $\mathfrak{D}$ has prime degree, and either $\mathfrak{B}_{0}$ splits $\mathfrak{D}$ or $\mathfrak{D}_{3_{0}}$ is a division algebra. In the latter case by Lemma 1 the algebra $\left(\mathfrak{D}_{\mathfrak{Z}_{0}}\right)_{\mathfrak{R}}=\mathfrak{D}_{\mathfrak{W}}$ is a division algebra, contrary to our hypothesis that $\mathfrak{W}$ splits $\mathfrak{D}$.

Since $\mathfrak{S}$ is a normal divisor of $(5)$ we have $T \mathfrak{S}=\mathfrak{S c} T, T S=S^{e} T$. If $e=1$, then the group $[T]$ is a normal divisor of $\$ S$, and $\oiint_{0}$ is cyclic of degree $p$ over $\Re$. By Lemmas 5 and 3 the algebra $\mathfrak{D}$ is cyclic. There remains the case $e>1$.

Now $T^{2} S=T S^{e} T=S^{e^{2}} T, \cdots, T^{m} S=S^{e^{m}} T^{m}=S=S^{e^{m}}$. Since $S$ has order $p$ we have

$$
e^{m} \equiv 1(\bmod p), \quad 0<e \leqq p-1 .
$$

We let $\nu$ be the least positive integer such that $e^{\nu} \equiv 1(\bmod p)$. Now $\nu \neq 1$, and $\nu$ must divide both $p-1$ and $m$. It follows that

$$
m=\nu q, \quad p-1=\mu \nu
$$

for integers $\mu$ and $q$. Notice that the group $[T]$ is not a normal divisor of $\$$, so that $乃_{0}$ is not a cyclic field over $\Re$.

By Lemmas 2, 5 the algebra $\mathfrak{D}$ has a subfield $\Omega$ of degree $p$ over $\Omega$ equivalent to $\bigcap_{0}$. Evidently $Z_{R}$ is equivalent to $\mathfrak{N}$, and $Z_{\Omega}=3 \times \Omega$. But the group of $\mathfrak{W}$ over $\mathbb{R}$ is $\mathfrak{S} ; \mathfrak{Z} \mathbb{R}$ is cyclic of degree $p$ over $\mathbb{R}$ with generating automorphism which we shall designate by $S$. Moreover if $z$ is in $\mathfrak{B}_{\mathfrak{R}}$, the automorphism $S$ which is given by $z \longleftrightarrow z^{S}$ goes into $z^{T} \longleftrightarrow\left(z^{S}\right)^{T}=\left(z^{T}\right)^{S^{e}}$ which is the automorphism $S^{e}$ of $\Re_{\Omega}$.

By Lemma 3 we have $\mathfrak{D}_{\mathfrak{R}}=\mathfrak{D} \times \mathfrak{R}=(\mathfrak{B}, S, g)$ for $g$ in $\mathfrak{R}$. This algebra has the automorphism

$$
d \longleftrightarrow d, \quad \lambda \longleftrightarrow \lambda^{T},
$$
$d$ in $\mathfrak{D}, \lambda$ in $\mathfrak{R}$. 
Apply this automorphism to $\mathfrak{D} \times \mathbb{R}$ and obtain

$$
\mathfrak{D} \times \mathfrak{R}=\left(\oiint_{\&}, S^{e}, g^{T}\right) \text {. }
$$

But then it is known that

$$
\mathfrak{D}=\left(\mathfrak{Z}^{\mathfrak{k}}, S,\left(g^{T}\right)^{f}\right) \sim\left(\Re_{\mathfrak{R}}, S, g^{T}\right)^{f},
$$

where $f$ is chosen so that $e f \equiv 1(\bmod p)$. It follows that

$$
\mathfrak{D} \sim\left(\Re, S, g^{T^{j}}\right)^{f^{j}}, \quad j=1,2, \cdots, n .
$$

We form $g_{0}=g g^{T^{\nu}} \cdots g^{T^{\nu(q-1)}}$ which is in the cyclic subfield $\Lambda$ of $\&$ of degree $\nu$ over $\Omega$. Now

(7) $\mathfrak{A}=\left(\mathfrak{马}_{\mathfrak{R}}, S, g\right) \times\left(\mathfrak{B}_{\mathfrak{R}}, S, g^{T^{\nu}}\right) \times \cdots \times\left(\mathfrak{Z}_{\mathfrak{R}}, S, g^{T^{\nu(q-1)}}\right) \sim\left(\mathfrak{Z}_{\mathfrak{R}}, S, g_{0}\right)$ over $\mathfrak{R}$. But $\mathfrak{A} \sim\left(\mathfrak{D}_{\mathfrak{R}}\right)^{\alpha}$, where by (6) we have

$$
\alpha=1+f^{\nu}+f^{2 \nu}+\cdots+f^{(q-1) \nu} \equiv q(\bmod p),
$$

since $e^{\nu} \equiv 1(\bmod p)$, ef $\equiv 1(\bmod p),(e f)^{\nu} \equiv f^{\nu} \equiv 1(\bmod p)$. Now $q$ is prime to $p$; hence $q q_{0} \equiv 1(\bmod p)$, and $\mathfrak{U} q_{0} \sim\left(\mathfrak{B}_{R}, S, g_{0} q_{0}\right) \sim\left(\mathfrak{D}^{q q_{0}}\right)_{\mathfrak{R}} \sim \mathfrak{D}_{\mathbb{R}}$, where $g_{0} q_{0}$ is in $\Lambda$. It follows that there is no loss of generality if we assume that $g$ is in $\Lambda$. We shall make this assumption.

By (6) we have

$$
\begin{aligned}
& \left(\mathfrak{D}_{\mathfrak{R}}\right)^{\nu} \sim\left(\mathfrak{B}_{\mathfrak{R}}, S, g\right) \times\left(\mathfrak{B}_{\mathfrak{L}}, S, g^{T}\right)^{f} \times \cdots \\
& \times\left(\Re_{R}, S, g^{r^{\nu-1}}\right)^{f^{\nu-1}} \sim\left(\Re_{\Re}, S, \gamma_{0}\right),
\end{aligned}
$$

where

$$
\gamma_{0}=\prod_{k=1}^{\nu}\left(g^{T^{k}}\right)^{f^{k}}
$$

But then

$$
\gamma_{0}^{T}=\prod_{k=1}^{\nu}\left(g^{T^{k+1}}\right)^{f k}, \gamma_{0}^{e}=\prod_{k=1}^{\nu}\left(g^{T^{k}}\right)^{e f^{k}}
$$

Since $e f \equiv 1(\bmod p)$ we have

$$
\gamma_{0}^{T}=\lambda_{0}{ }^{p} \gamma_{0}^{e}, \quad \lambda_{0} \text { in } \Lambda .
$$

Now $\nu \nu_{0} \equiv 1(\bmod p)$ and $\left(\mathfrak{D}_{\mathfrak{R}}\right)^{\nu \nu_{0}} \sim \mathfrak{D}_{\mathfrak{R}} \sim\left(\mathfrak{Z}_{\mathfrak{R}}, S, \gamma_{1}\right)$, where $\gamma_{1}=\gamma_{0}{ }^{\nu_{0}}$, and (12) implies that

$$
\gamma_{1}^{T}=\lambda^{p} \gamma_{1}^{e}, \quad \lambda \text { in } \Lambda .
$$

Since $\mathfrak{D}_{\mathfrak{R}}$ and $\left(\mathfrak{B}_{\mathfrak{R}}, S, \gamma_{1}\right)$ have the same order, they are equivalent, and we have proved the following lemma: 
LEMma 6. The algebra $\mathfrak{D}_{\mathfrak{l}}$ has the generation $\mathfrak{D}_{\mathfrak{R}}=\left(\mathfrak{Z}_{\mathfrak{R}}, S, \gamma\right)$ where $\gamma_{1}$ is in $\Lambda$ and (13) holds.

The cyclic algebra $\mathfrak{D}_{\mathfrak{R}}$ contains a quantity $y_{0}$ such that $y_{0}{ }^{p}=\gamma_{1}$, and $\mathfrak{R}\left(y_{0}\right)$ is a maximal subfield of $\mathfrak{D}_{\mathfrak{R}}$. Hence $\mathfrak{R}\left(y_{1}\right) \cong \mathbb{R}\left(y_{0}\right)$ is a scalar splitting field of $\mathfrak{D}_{\mathfrak{R}}$. But by (13) we have

$$
\gamma_{1}^{T^{j}}=\lambda_{j}^{p} \gamma_{1}^{e^{j}}, \quad j=0,1, \cdots, \nu-1 ;
$$

and if

$$
y=y_{1}+\lambda_{1} y_{1}^{e}+\lambda_{2} y_{1} e^{2}+\cdots+\lambda_{\nu-1} y_{1}^{e^{\nu-1}},
$$

then $\mathfrak{R}\left(y_{1}\right)=\mathfrak{R}(y)$. For $0<e \leqq p-1, e^{i} \equiv e^{j}(\bmod p)$ if and only if $i-j$ is divisible by $\nu ; y$ is clearly not in $\mathbb{R}$, and $y$ in $\mathfrak{R}\left(y_{1}\right)$ generates $R\left(y_{1}\right)$. It follows that $\mathfrak{R}(y)$ splits $\mathfrak{D}_{\mathfrak{R}}$. But $\Re$ has characteristic $p$ and

$$
y^{p}=\gamma_{1}+\gamma_{1}^{T}+\cdots+\gamma_{1}^{T^{\nu-1}}=\gamma \text { in } \Omega .
$$

Now $\mathscr{L}(y)=[\Re(y)]_{\Re}$, and $\Omega(y)$ splits $\mathfrak{D}$ by the proof of Lemma 5 . By Lemma $4, \mathfrak{D}$ is a cyclic algebra.

In closing let us note that all of our proof is valid for arbitrary fields except the final result (16), which depends essentially* upon the property that $\Omega$ has characteristic $p$.

The University of Chicago

* Added in proof: When $p=3$ we may replace (13) by $\gamma_{1}^{T}=\gamma_{1}{ }^{-1}$, and direct computation shows that if $a$ is in 8 with trace zero and norm $\alpha$, and $u=a\left(1+y_{1}+y_{1}^{-1}\right)$, then $u^{3}=\alpha\left(2+\gamma_{1}+\gamma_{1}^{T}\right)$ in $\Re$. This proves $\mathfrak{D}$ cyclic for any characteristic. 